\title{
$\underline{\text { Research and Clinical Applications of Assessing Balance }}$
}

\author{
By: Kevin M. Guskiewicz and David H. Perrin*
}

Guskiewicz, K.M., \& Perrin, D.H. (1996). Research and clinical applications of assessing balance. Journal of Sport Rehabilitation, 5:45-63.

\section{***Note: Figures may be missing from this format of the document}

\begin{abstract}
:
Returning athletes to competition following injury often creates a dilemma for athletic trainers and team physicians. Most clinicians gather as much data as possible before deciding whether to return an athlete to competition following injury. The status of the postural control system and balance is important for certain pathologies and therefore should be considered in these clinical decisions. As more high-tech balance systems become available, it is important for clinicians to understand not only what is available but what these devices measure. This paper will review the relationship between the postural control system and the kinetic chain, traditional and contemporary techniques for assessing balance, and ways in which clinicians can bridge the gap between balance research and clinical practice.
\end{abstract}

\section{Article:}

Although a seemingly simple task, maintaining equilibrium or balance while standing upright is an important motor skill. Balance, as described by Nashner (41), is a complex process involving coordination of multiple sensory, motor, and biomechanical components. An individual senses the position of his or her body in relation to gravity and the surroundings by combining visual, vestibular, and somatosensory inputs (41). Balance movements also involve motions of the ankle, knee, and hip joints, which are controlled by coordinated actions along the kinetic chain (Figure 1). These processes are all vital for producing fluid sport-related movements.

\section{Postural Control System}

The postural control system operates as a feedback control circuit between the brain and the musculoskeletal system. The musculature of the legs, feet, and truncus, using this feedback circuit, allows the individual to stand erect against the forces of gravity $(22,31,38,55)$. Postural stability is also greatly influenced by factors such as nervous disorders, dysfunction of optic nerve and vestibular mechanisms, fatigue, and mental status (51).

\footnotetext{
${ }^{*}$ Kevin M. Guskiewicz is with the Department of Physical Education, Exercise and Sport Science, The University of North Carolina at Chapel Hill, Chapel Hill, NC 275998700. David H. Perrin is with Athletic Training Education and Research, Curry School of Education, University of Virginia, Charlottesville, VA 22903.
} 


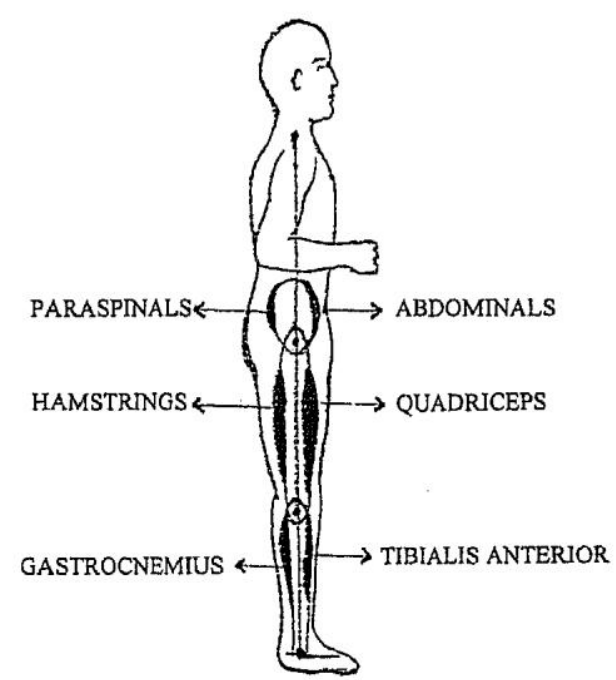

Figure 1-Major joints and muscles responsible for controlling movements of thı body's center of gravity (COG) during standing balance. (Reproduced with permis sion from NeuroCom International, Inc.)

Feedback obtained from the vestibular, visual, and proprioceptive (somatosensory) sensors relays commands to the muscles of the extremities, which then generate an appropriate contraction to maintain postural stability $(26,31,38,50,51)$. The vestibular apparatus is the organ responsible for detecting sensations concerned with equilibrium. The apparatus is composed of a system of bony tubes and chambers within the temporal bone called the bony labyrinth. The labyrinth is adjacent to and continuous with the cochlear duct of the inner ear and also consists of three semicircular canals and two large chambers known as the utricle and the saccule $(22,55)$.

Information from the vestibular apparatus can be used in three different ways. First, the information is used to control eye muscles so that when the head changes position, the eyes can stay fixed on one point. Second, vestibular information can be used to maintain upright posture. The vestibular organs are often referred to as the "sense organs of balance," despite research findings to the contrary. That is, very few postural reflexes rely primarily on vestibular input (28, $38,43,52)$. A third use of vestibular information involves conscious awareness of the body's position and acceleration after Information has been relayed to the cortex by the thalamus (55).

Investigators $(8,28,38,43)$ agree that the vestibular system is primarily involved in stabilizing slow body sway, which is achieved by a much lower level of leg activation. Much of this research suggests that people rely mostly on visual and somatosensory inputs when maintaining balance under normal conditions. When sudden changes or perturbations are induced causing a person to change his or her direction of movement or head position (i.e., leaning the head sideways, forward, or backward), the automatic control mechanism provided by vestibular input becomes crucial for stabilizing the direction of gaze and ultimately one's equilibrium. Vision is obviously very important for maintaining control of balance, especially under conditions of postural perturbation. Moreover, the eyes would be of little use in detecting an image if they did not remain fixed on an object long enough to gain a clear image. Therefore, when the head is suddenly tilted, signals from the semicircular canals cause the eyes to rotate in an equal and 
opposite direction to the rotation of the head (22). This is a function of the vestibulo-ocular reflex. Thus, when both the support surface and visual surroundings are tilted, the vestibular input automatically takes precedence (38). In short, the vestibular apparatus mainly contributes to posture by maintaining reflexes associated with keeping the head and neck in the vertical position and allowing the vestibulo-ocular reflex to control eye movement.

The proprioceptive system functions via the mechanoreceptive senses of touch, pressure, vibration, and tickle, all of which are commonly referred to as the tactile senses, and the sense of position, which determines the relative positions and rates of movement of parts of the body (22, $55)$.

Muscle spindles and Golgi tendon sensory receptors (proprioceptors) play a vital role in the nervous system's control of posture. They provide the nervous system with continuous feedback about the status of each muscle. Muscle length and changes in length are monitored by stretch receptors embedded within the muscle. These receptors consist of afferent nerve fiber endings that are wrapped around modified muscle fibers, several of which are enclosed in a connectivetissue capsule. The entire structure is called a muscle spindle $(22,55)$. Muscle spindles send information to the nervous system about either the muscle length or its rate of length. When afferent fibers from the muscle spindle enter the central nervous system, they divide into branches that can take several different paths. One path directly stimulates motor neurons going back to the muscle that was stretched, thereby completing a reflex arc known as the stretch reflex or myotatic reflex. This reflex causes a muscle contraction in response to a muscle being stretched (55).

Golgi tendon organs (GTO) located in the tendons near their junction with the muscles serve as a second type of afferent receptor (proprioceptor). They are responsible for sending information about tension in the muscle or rate of change of tension $(22,55)$. The afferent neuron's firing activity supplies the motor- control systems (both locally and in the brain) with continuous information about muscle tension. The GTO is designed to serve as a protective mechanism to relax a muscle that is being overstretched. It senses tension within a muscle, transmits the information to the central nervous system, and through polysynaptic reflexes inhibits the motor neurons of the contracting muscle (55),

Ankle rotation is the most probable stimulus of the functional stretch reflex (myotatic) that occurs in many persons. It appears to be the first useful phase of activity in the leg muscles after a change in erect posture (37). The myotatic reflex can be seen when perturbations of gait or posture automatically evoke functionally directed responses in the leg muscles to compensate for imbalance or increased postural sway $(8,37)$, Muscle spindles sense a stretching of the agonist, thus sending information along its afferent fibers to the spinal cord. There the information is transferred to alpha and gamma motor neurons that carry information back to the muscle fibers and muscle spindle, respectively, and contract the muscle to prevent or control additional postural sway (8).

\section{Isolation of Sensory Input}

From our knowledge of the central nervous system's involvement in maintaining upright posture, we can divide the process into two components. "Sensory organization," as described by Nashner 
(38), involves processes that determine the timing, direction, and amplitude of corrective postural actions based upon information obtained from the vestibular, visual, and somatosensory (proprioceptive) inputs. Despite the availability of multiple sensory inputs, the central nervous system generally relies on only one sense at a time for orientation information. For healthy adults, the preferred sense for balance control comes from somatosensory information (e.g., feet in contact with the support surface) (38). The second component, "muscle coordination," describes processes that determine the temporal sequencing and distribution of contractile activity among the muscles of the legs and trunk which generate supportive reactions for maintaining balance. Research suggests that balance deficiencies in people with neurological problems can result from inappropriate interaction among the three sensory inputs that provide orientation information to the postural control system. A patient may be inappropriately dependent on one sense for situations presenting intersensory conflict $(38,50)$.

Several studies have attempted to isolate and clarify which sensory inputs are most involved with regulating posture and how the interaction among these inputs affects posture control $(7,8,10$, $28,42,46)$. Shumway-Cook and Horak (50) introduced a technique to systematically remove or conflict sensory input from one or more of the three senses. The technique, referred to as the Clinical Test of Sensory Interaction and Balance (CTSIB), or "Foam and Dome Test," uses combinations of three visual and two support-surface conditions during assessment of postural sway (Figure 2). A reference point on the inside of the visual-conflict dome moves in phase with the subject's head movement, therefore altering vestibular input. Thus, increased postural instability while wearing the visual-conflict dome (Condition 3 in Figure 2) suggests abnormal reliance on vision for posture control, which is common with patients having postconcussion vestibular syndrome or benign paroxysmal positional nystagmus (38). The technique to conflict sensory imput involves alteration of the support surfaces. The conditions include a hard, flat surface that ensures accurate orientation information from the somatosensory system, and a compliant section of medium-density foam that reduces the accuracy of the orientation information. Increased postural instability under conditions where proprioceptors of the feet are eliminated (Conditions 4-6) suggests that the other sensory modalities (vestibular and/or visual) are not adequately compensating for the loss of proprioception.

Another technique of isolating sensory modality has been proposed by Nashner (40). Sensory Organization Testing (SOT) evaluates the integrity of the three sensory modalities by selectively disrupting somatosensory and/or visual information regarding body center of gravity (COG) orientation in relation to vertical and then measuring the patient's ability to maintain balance. Sway referencing involves tilting the support surface and/or the visual surround (wall) in an anterior - posterior direction according to the patient's COG sway (Figure 3). Under swayreferenced conditions, the orientation of the support surface and/or the visual surround remains constant in relation to the COG sway angle $(40,43)$. The theory behind sway referencing is similar to that proposed by Shumway- 


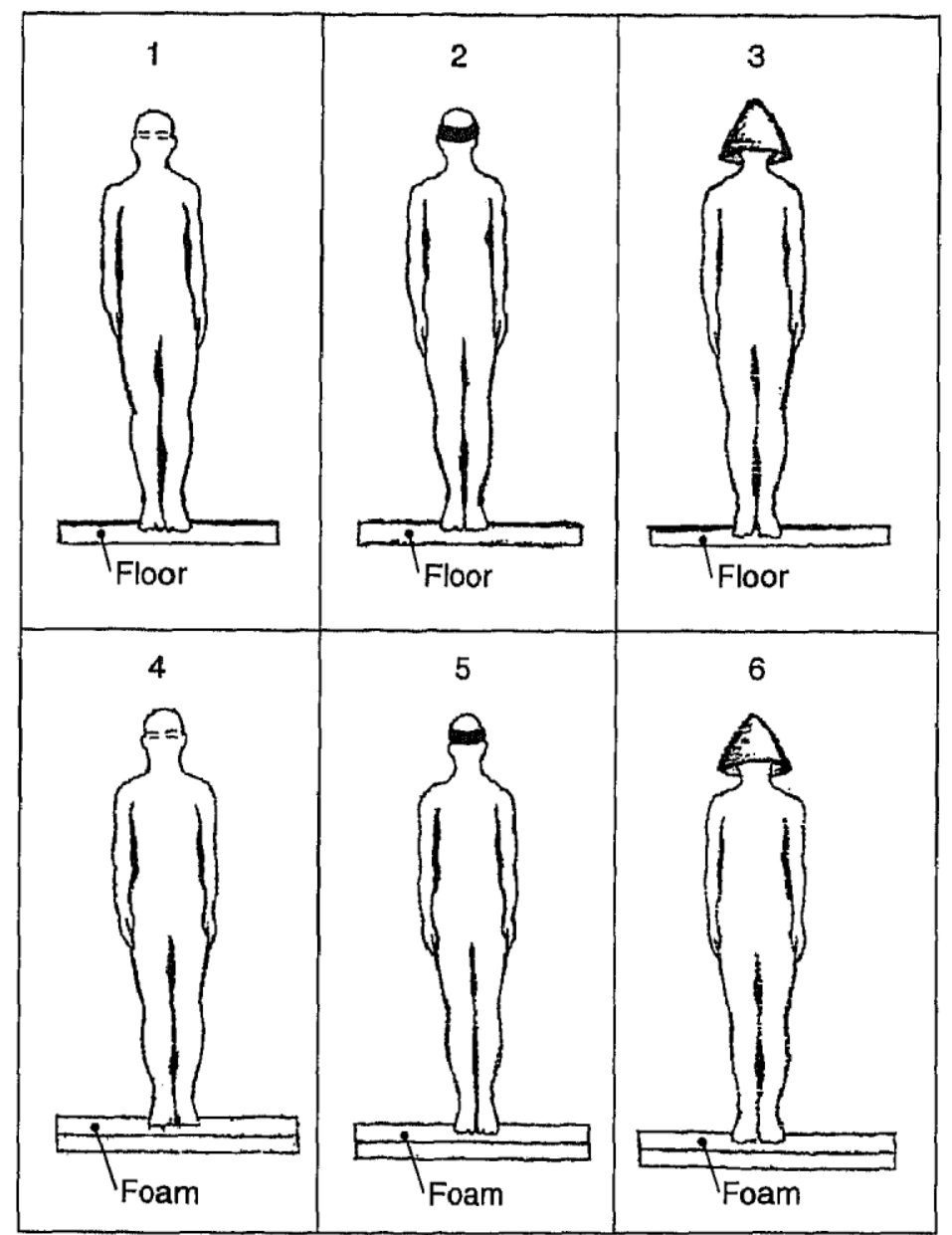

Figure 2-Six testing conditions for Clinical Test of Sensory Organization and Balance as described by Shumway-Cook and Horak (50).

Cook and Horak (50); healthy subjects should ignore a sway-referenced sensory input that is functionally inaccurate and maintain balance using other sensory inputs. In addition to sway referencing, eyes closed conditions are used to further isolate somatosensory and vestibular systems. Sensory analysis can then be performed comparing the six test conditions. For example, a vestibular ratio can be calculated by comparing Condition 5 to Condition $1(5 ; 1)$. A low score would suggest that a patient makes poor use of vestibular cues or that vestibular cues are unavailable.

\section{Balance in Relation to the Closed Kinetic Chain}

Balance is the process of maintaining the COG within the body's base of support. The human body is a very tall structure balanced on a relatively small base, and 


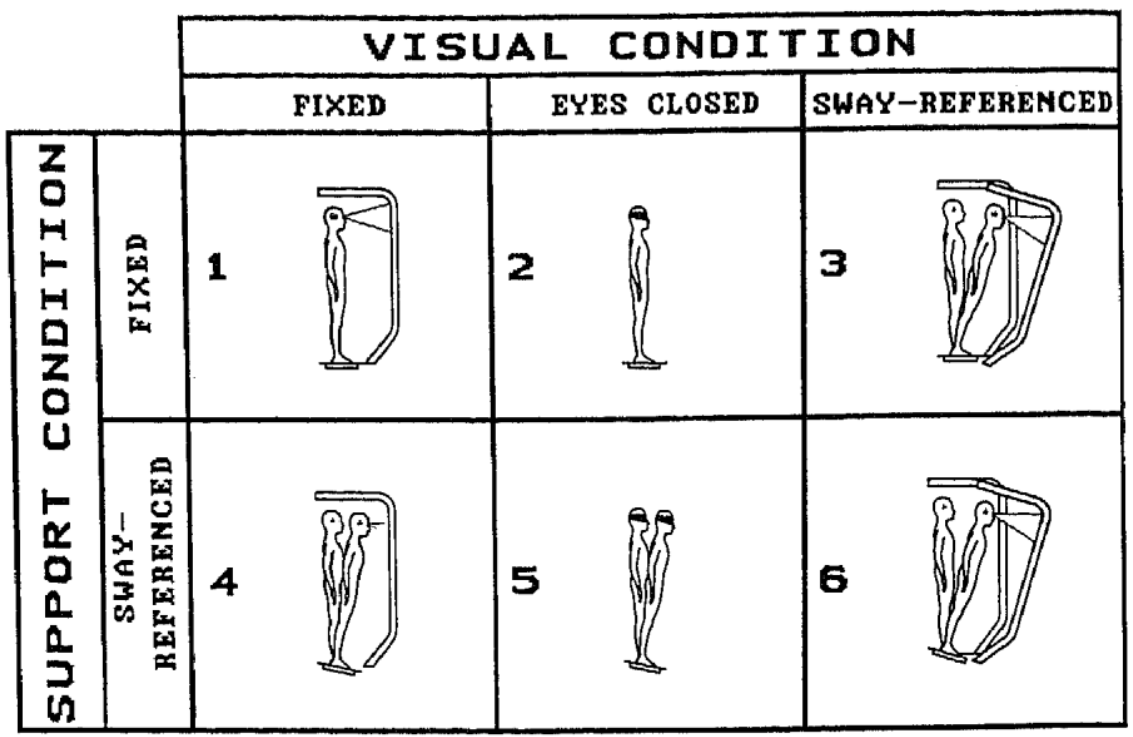

Figure 3-Six testing conditions for Sensory Organization Test used with Neuro* Com's EquiTest system. (Reproduced with permission from NeuroCom International, Inc.)

its center of gravity is quite high, being just above the pelvis (55). Many factors enter into balance control within this designated area. One component often overlooked is the role balance plays within the kinetic chain. Ongoing debates as to how the kinetic chain should be defined and whether open or closed kinetic chain exercises are best have caused many clinicians to lose sight of what is most important. An understanding of the postural control system as well as the theory of the kinetic (segmental) chain about the lower extremity helps us conceptualize the role of the chain in maintaining balance. Within the kinetic chain, each moving segment transmits forces to every other segment along the chain, and a segment's motions are influenced by forces transmitted from other segments (6). Maintaining equilibrium or balance is associated with the closed kinetic chain, as the distal segment (foot) is fixed beneath the base of support.

Coordination of automatic postural movements during the act of balancing is not determined solely by the muscles acting directly about the joint. Leg and trunk muscles exert indirect forces on neighboring joints through inertial interaction forces among body segments $(39,44)$. A combination of one or more strategies (ankle, knee, hip) is used to move the COG back to a stable or balanced position when a person's balance is disrupted by an external perturbation. Injury to any one of the joints or corresponding muscles along the kinetic chain can result in a loss of appropriate feedback for maintaining balance.

\section{Measurement of Balance}

Several methods of balance assessment have been proposed for clinical use. Many of the techniques have been criticized for offering only subjective information or a "qualitative" measurement of balance rather than an objective or "quantitative" measure.

\section{Subjective Balance Assessment}

Prior to the mid-1980s, there were very few methods for systematic and controlled assessment of balance in the sports medicine setting. Static balance in athletes has traditionally been assessed 
with the standing Romberg test. For this test, the subject stands with feet together, arms at the side, and eyes closed. Normally a person can stand motionless in this position, but a tendency to sway or fall to one side is considered to indicate a loss of proprioception (4). The Romberg test has been criticized for its lack of sensitivity and objectivity. It is considered to be a rather qualitative assessment of static balance, because a considerable amount of stress is required to make the subject sway enough for an observer to characterize the sway (31). Other tests of static balance include a single-leg stance test, which includes balancing on one leg for a specified amount of time with eyes open or closed. The tandem Romberg test (14) requires placement of one foot in front of the other (heel to toe).

Dynamic balance can be assessed through functional reach tests, timed agility tests such as the figure eight test $(9,14,56)$, carioca or hop tests $(30,56)$, and balance beam walking with the eyes open or closed. The objective in most of these tests is to decrease the size of the base of support, in an attempt to determine an athlete's ability to control upright posture while moving. Many of these tests have failed to quantify balance adequately, as they merely report the time that a particular posture is maintained, angular displacement, or the distance covered after walking $(3,15,45,56)$.

\section{Objective Balance Assessment}

More recently, advancements in technology have provided the medical community with force platform systems for quantitatively assessing both static and dynamic balance. These systems provide an easy, practical, and sometimes cost- effective method of quantitatively assessing functional balance through analysis of postural sway. Thus, the potential exists to assess injured athletes and (a) identify possible abnormalities that might be associated with injury, (b) isolate various systems that are affected, and (c) develop recovery curves based on quantitative measures for determining readiness to return to activity. Following are some high-technology balance systems.

Static systems

Chattecx Balance System

EquiTest

Force Plate

Pro Balance Master

Smart Balance Master
Dynamic systems

Biodex Stability System

Chattecx Balance System

EquiTest

EquiTest with EMG

Force Plate

Kinesthetic Ability Trainer (KAT)

Pro Balance Master

Smart Balance Master 


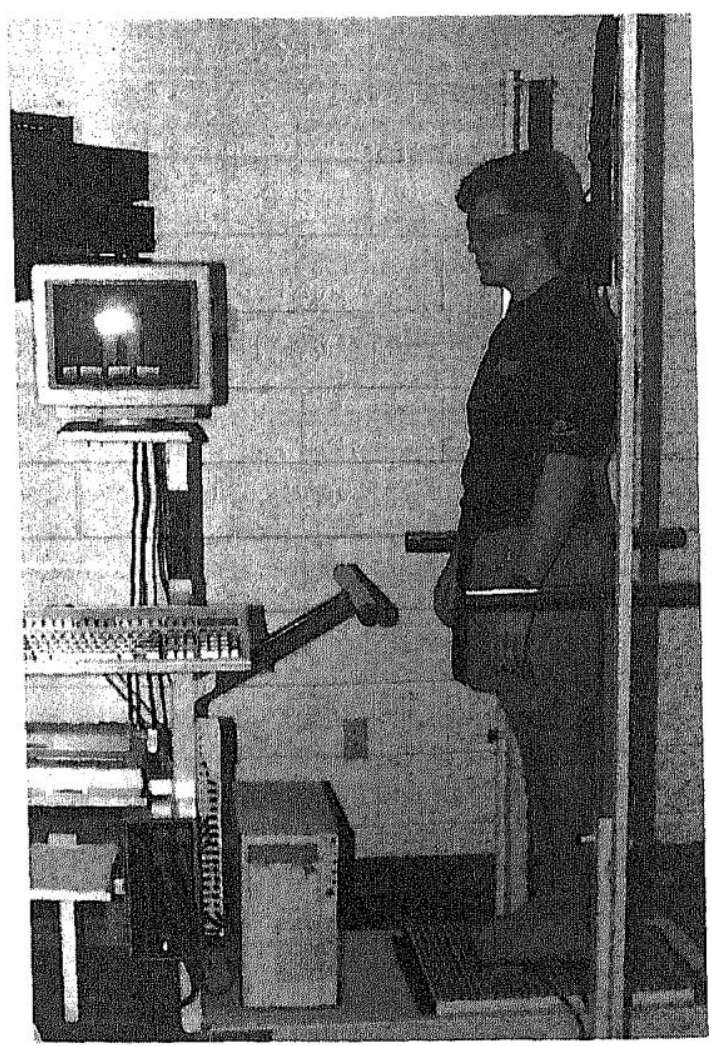

Figure 4 - Chattecx Balance System.

A basic force plate consists of a flat, rigid surface supported on three or more points by independent force-measuring devices, As the athlete stands on the force plate, the position of the center of vertical forces exerted on the force plate over time is calculated. The center of vertical force movements provides an indirect measure of postural sway (40). The Kistler force plate was used for much of the early research in postural stability and balance $(3,11,19,34,36)$. Manufacturers such as Chattecx Corporation (Nixon, TN) and NeuroCom International, Inc. (Clackamas, OR) have developed more sophisticated systems with expanded diagnostic and training capabilities (Figures 4 and 5). Biodex Medical Systems (Shirley, NY) recently developed a dynamic multiaxial tilting platform that offers computer-generated data similar to that of a force plate system (Figure 6).

Force platforms ideally evaluate four aspects of postural control: steadiness, symmetry, dynamic stability, and dynamic balance. Steadiness is the ability to keep the body as motionless as possible. This is also considered a measure of postural sway. Symmetry is the ability to distribute weight evenly between the two feet in an upright stance. This is a measure of center of pressure $(\mathrm{COP})$, center of balance $(\mathrm{COB})$, or center of force $(\mathrm{COF})$, depending on which testing 


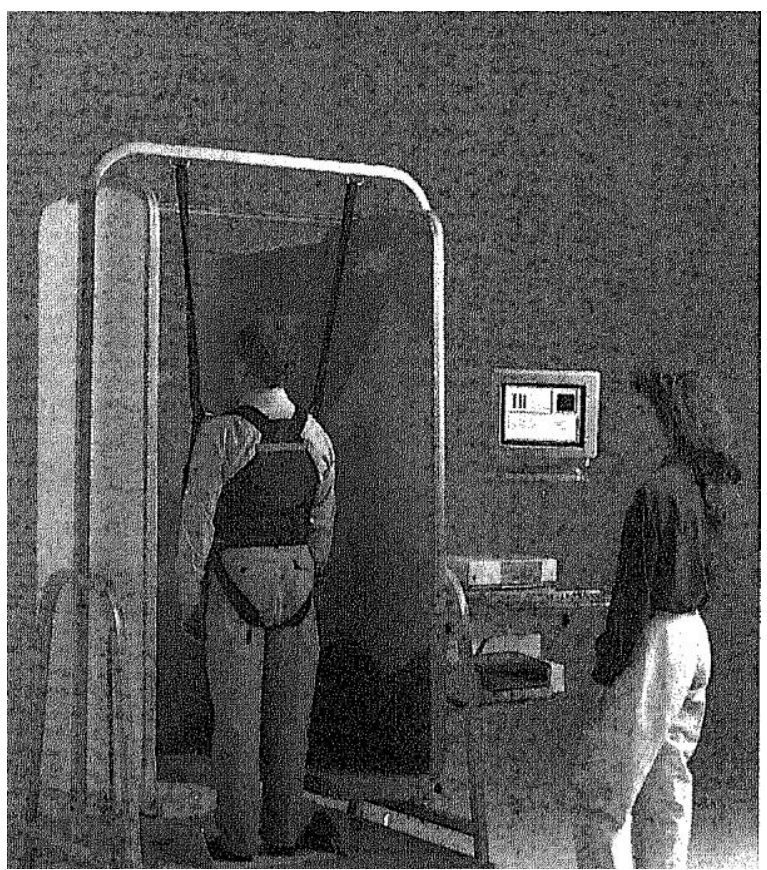

Figure 5 - EquíTest system.

system is used. Dynamic stability is the ability to transfer the vertical projection of the COG around a stationary supporting base (19), This is often referred to as a measure of one's perception of "safe" limits of stability, as the goal is to lean or reach as far as possible without losing one's balance, Dynamic balance assessment is somewhat different, in that the tester is measuring postural responses to external perturbations from a platform moving in one of four directions: tilting toes up, tilting toes down, shifting medial-lateral $(\mathrm{M}-\mathrm{L})$, and shifting anterior-posterior $(\mathrm{A}-\mathrm{P})$. Platform perturbation on some systems (Pro Balance Master, EquiTest) is unpredictable and is determined by the positioning and sway movement of the subject. In such cases, a person's reaction response can be determined. Other systems (Chattecx Balance System) have a more predictable sinusoidal waveform, which remains constant regardless of subject positioning.

Many of these force platform systems measure the vertical ground reaction force and provide a means of computing the COP. The COP represents the center of distribution of the total force applied to the supporting surface. The COP is calculated from horizontal moment and vertical force data generated by triaxial force platforms. The COB, in the case of the Chattecx Balance System, is the 


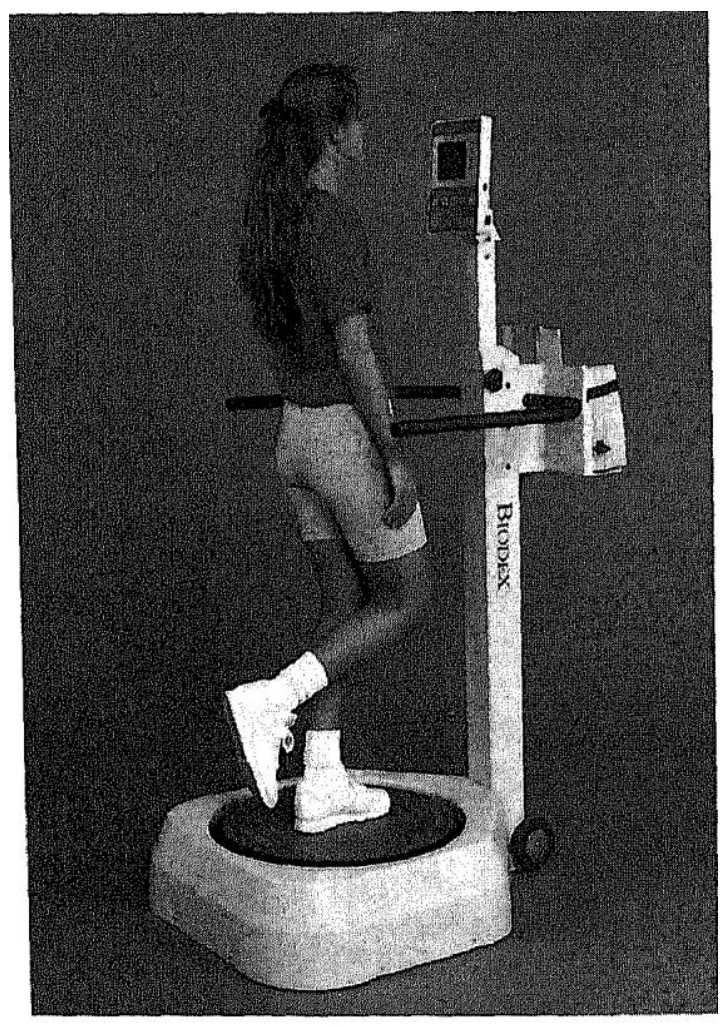

3iodex Stability System.

point between the feet where the ball and heel of each foot have $25 \%$ of the body weight. This point is referred to as the relative weight positioning over the four load cells as measured only by vertical forces. The COF, on NeuroCom's EquiTest, is the center of the vertical force exerted by the feet against the support surface. In any case (COP, COB, COF), the total force applied to the force platform fluctuates because it includes both body weight and the inertial effects of the slightest movement of the body (which occurs even when one attempts to stand motionless). It is theorized that the movement of these force-based reference points varies according to the movement of the body's COG and the distribution of muscle forces required to control posture. Ideally, healthy athletes should maintain their $\mathrm{COP}$ very near the $\mathrm{A}-\mathrm{P}$ and $\mathrm{M}-\mathrm{L}$ midlines.

Once the COP, COB, or COF is calculated, several other balance parameters can be attained. Deviation from this point in any direction represents a person's postural sway. Postural sway can be measured in various ways, depending on which system is used. Mean displacement, length of sway path, length of sway area, amplitude, frequency, and direction with respect to the COP can be calculated on most systems. An equilibrium score, comparing the angular difference between the calculated maximum anterior to posterior COG displacements to a theoretical maximum displacement, is unique to NeuroCom International's EquiTest. Sway index (SI), representing the degree of scatter of data about the COB, is unique to the Chattecx Balance System.

Force plate technology, such as NeuroCom International's EquiTest, the Pro and Smart Balance Master systems, and Chattanooga Group's Balance System, allow for quantitative analysis and understanding of a subject's postural instability. These systems are fully integrated with hardware/software for quickly and quantitatively assessing and rehabilitating balance disorders. 
These systems allow for both static and dynamic balance assessment in either double- or singleleg stances, with eyes open or eyes closed. NeuroCom's EquiTest system is equipped with a moving visual surround (wall), the most sophisticated technology available for isolating and assessing sensory modality interaction. While the EquiTest is most useful for assessing neurological pathologies, the Pro and Smart Balance Master systems are most useful for assessing and rehabilitating orthopedic injuries.

The Kinesthetic Ability Trainer, or KAT (Greg, Inc., Vista, CA), and the Biodex Stability System (Biodex Medical Systems, Shirley, NY) both utilize a dynamic multiaxial platform similar to that of a BAPS board or wobble board used for lower extremity rehabilitation. These platforms allow approximately $20^{\circ}$ of deflection in any direction and are interfaced with computer software. It is theorized that this degree of deflection is sufficient to stress joint mechanoreceptors, which provide proprioceptive feedback (at end ranges of motion) necessary for balance control. Clinicians can therefore assess deficits in dynamic muscular control of posture relative to joint pathology. The Biodex Stability System (Figure 6) measures the patient's ability to control the platform's angle of tilt. Tilt is quantified as a variance from center, as well as degrees of deflection over time, at various stability levels. A large variance indicates poor muscle response.

\section{Clinical Applications of Balance Assessment}

Assessment of balance and postural control has traditionally focused on the geriatric population rather than an athletic population. However, recent trends in the diagnosis and rehabilitation of athletic injuries have placed more emphasis on balance $(11,20,21,27,30)$. While some pathologies have little or no connection to balance, others are highly implicated.

\section{Head Injury}

It has been suggested that changes in simple motor skills $(23,32)$ and postural control $(1,29)$ accompany mild head injury. However, the tests used to assess these injuries are often criticized for their lack of sensitivity and objectivity.

Neurological status following mild head injury has been assessed using balance as a criterion variable. Athletic trainers and team physicians have long evaluated head injuries with the Romberg test of sensory modality function to test "balance." This is an easy and effective sideline test; however, the literature suggests there is more to posture control than just balance and sensory modality $(37,38,42,43,46,50)$, especially when assessing people with head injury (29). The postural control system, which is responsible for linking brain to body communication, is often affected by mild head injury.

Related Research. Despite the common dysfunction of cerebral and/or vestibular mechanisms accompanying head injury, very few studies have focused on the effects of head injury on postural equilibrium. Of the studies that have been conducted, most have focused on severely injured or chronically injured patients. Arcan et al. (1) found that subjects with hemiplegia or craniocerebral injuries who were tested on a force platform at the beginning of their rehabilitation period demonstrated abnormal weight shifting, suggesting disruption to one or all of the mechanisms responsible for controlling equilibrium. In an attempt to better understand the role of the cerebellum in posture, Mauritz, Dichgans, and Hufschmidt (34) studied postural sway 
in three groups of subjects with restricted cerebellar lesions. Patients with anterior lobe lesions demonstrated a specific tremor in the A-P direction. $\mathrm{M}-\mathrm{L}$ sway was less characteristic and smaller in amplitude, although patients with uncompensated vestibular lesions showed marked lateral sway and eventually fell, as did patients with vestibulocerebellar lesions. Finally, Ingersoll and Armstrong (29) used a force platform and the six testing conditions for isolating sensory input (Figure 2) to monitor translation of COP in 48 subjects who were at least 1 year postinjury. Results revealed that severely head-injured subjects (unconscious for $>6 \mathrm{hr}$ ) demonstrated greater $\mathrm{A}-\mathrm{P}$ and $\mathrm{M}-\mathrm{L}$ sway than mildly head-injured or normal subjects. Although total sway did not differ between groups, head-injured subjects maintained their COP at greater distances from the center of their base of support. Furthermore, these subjects made fewer postural corrections, especially when one or more of the sensory modalities were conflicted or eliminated (29).

Guskiewicz and Perrin (20) studied postural stability in 10 college and high school football players who had suffered mild head injury (MHI). The Chattecx Balance System (Chattanooga Group, Hixon, TN) was used under a modified protocol for isolation of sensory input. The six tests described in Figure 2 were used, and the three eye conditions were repeated while subjects were on a tilting platform. Results revealed that MHI subjects demonstrated significant increases in postural sway on Day 1 postinjury as compared to the prescreening and/or subsequent tests (Figure 7). Furthermore, MHI subjects had significant improvements from Day 1 postinjury to Day 3 postinjury when compared with

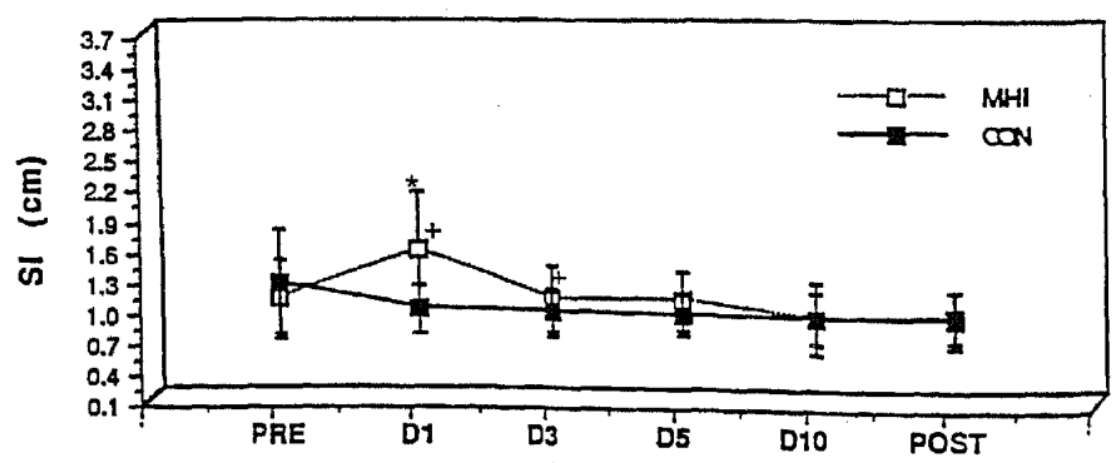

Figure 7 - Mild head-injured subjects demonstrated increased postural sway 1 day following injury, compared to control subjects (20). 'Significantly different from preceding test. *Significantly different from other group. Tukey HSD $=.30, p<.05$.

control subjects, who demonstrated insignificant changes. These findings suggest that computerized dynamic posturography is a useful tool in objectively assessing postural stability in MHI subjects. Subjects with MHI appear to demonstrate impaired postural stability 1 to 3 days following injury. This information should aid clinicians in deciding when an athlete can safely return to participation.

\section{Musculoskeletal Injury}

It is commonly believed that musculoskeletal injury to the lower extremity alters the somatosensory (proprioceptive) input that is essential for neuromuscular coordination. Joint proprioceptors are believed to be damaged during both complete and incomplete rupture of the lateral ligaments of the ankle because the joint receptor fibers possess less tensile strength than the ligament fibers (17). Damage to the joint receptors is believed to cause joint deafferentation, 
which diminishes the supply of messages from the injured joint up the afferent pathway and disrupts proprioceptive function (17). Furthermore, when the anterior cruciate ligament (ACL) in the knee is torn or stretched, kinesthesia of the knee joint is decreased. It is believed that a patient's ability to balance on the ACL-injured leg may be decreased, even following surgical reconstruction of the knee $(2,47)$. Current rehabilitation protocols for the lower extremity emphasize the importance of balance and proprioceptive exercises $(30,33)$, although there is a lack of consistent findings on balance assessment of the injured athlete.

Related Research. Since 1965, Freeman (16) has theorized that if ankle injuries cause partial deafferentation and functional instability, a person's postural sway should be altered due to a proprioception deficit. While some studies $(53,54)$ have not supported Freeman's theory, other more recent studies $(5,21,48)$ using high-tech equipment have revealed that subjects with ankle sprains demonstrate increases in postural sway compared to uninjured subjects.

Guskiewicz and Perrin (21) investigated 13 subjects with acute inversion ankle sprains and 12 uninjured subjects for postural sway on the Balance System (Chattecx Corporation, Chattanooga, TN) under two treatment conditions (orthotic, nonorthotic) and four platform movements (stable, inversion/eversion, plantar flexion/dorsiflexion, medial/lateral glides). Results revealed that subjects with ankle injuries swayed more than uninjured people when assessed on a single-leg test. The analysis also revealed that custom-fit orthotics may restrict undesirable motion at the foot and ankle and may enhance abilities of joint mechanoreceptors to detect perturbations and provide structural support for injured ankles.

Several studies have used force plate technology to evaluate the effects of ACL ruptures on standing balance. While some studies have revealed balance deficits in ACL-deficient subjects $(18,35)$, others have not $(13,24)$. Thus, there appear to be conflicting results from these studies depending on which parameters are measured. Mizuta et al. (35) found significant differences in postural sway (COP length of movement and sway distance) between 11 functionally stable and 15 functionally unstable subjects who had unilateral. ACL-deficient knees, Faculjak et al. (13), however, found no differences in postural sway between 8 ACL-deficient subjects and 10 normal subjects (average latency and response strength) when tested on the EquiTest system.

\section{Research Applications of Balance Assessment}

Researchers have studied many of the aforementioned balance parameters in an attempt to establish normative data. It is important for clinicians to understand and use normative data in setting baselines for their patients. The effects of height, weight, sex, age, stance, and vision on static balance in normal subjects have been studied by several investigators using force platforms. There appears to be agreement among investigators on age, stance, and vision; however, results are inconsistent on height, weight, and sex.

Ekdahl et al. (11) attempted to obtain stabilometric reference values for healthy males and females of various age groups ranging from 20 to 64 years. The study utilized an AMTI force platform and Computer Automated Stability Analysis software to calculate the COP and sway amplitude from the mean position in the lateral and sagittal directions. The results revealed that males demonstrated greater sway than females, older subjects demonstrated greater sway than younger ones, and attempts to balance on one leg and/or blindfolded increased postural sway. 
Significant correlations were found between age and length of sway path for one-leg standing ( $\mathrm{r}$ $=.40$ for right leg and $\mathrm{r}=.48$ for left leg; $\mathrm{p}<.001$ ). Ekdahl et al. found no significant correlations between length of sway path and height and weight, although others $(12,51)$ found height and weight to correlate with postural sway.

Era and Heikkinen (12) reported that postural sway was two- to threefold greater when the eyes were closed than when they were open, and that the increase in sway was more pronounced in the $\mathrm{A}-\mathrm{P}$ direction. Furthermore, postural sway was eight times greater during monopedal standing versus bipedal standing, and $\mathrm{M}-\mathrm{L}$ sway became as important as $\mathrm{A}-\mathrm{P}$ sway during monopedal standing. Significant correlations were reported between postural sway and percentage body fat $(r=.17$ for eyes open and $r=.21$ for eyes closed; $p<.05)$. Again, significant differences were found between age groups, with postural sway being more pronounced in the older groups.

In an attempt to address the clinical application of measuring body movement, Sugano and Takeya (51) compared the projection of COG of normal subjects to that of subjects who had pathology or were under the effect of manipulated conditions. Subjects were required to stand as still as possible with their eyes closed, as their projection of COG was measured on a four-point suspension system (force plate). Results revealed no correlation between body movement and age, although a trend was observed suggesting that the movement pattern was larger in old age as compared to youth. Significant correlations between body movement and body weight $(\mathrm{r}=.31, \mathrm{p}$ $<.01)$ and body height $(\mathrm{r}=.52, \mathrm{p}<.01)$ suggest that heavy and taller subjects tend to have a large body movement. The authors concluded that in general, body movement for women is smaller than for men and that open-eye conditions result in movement patterns that are two thirds the size of movement patterns for closed-eye conditions.

Normal balance performance research using force platforms has tracked COP movement within $0.01 \mathrm{~cm}$. Murray et al. (36) recorded the mean position of the COP, the average distance of the COP from its mean position in the fore - aft and left — right directions, and the total excursions of the COP in 24 healthy men divided into three groups according to age (younger, middle, older). The authors reported that vertical force constantly fluctuated above and below body weight in all of the tests performed (double-limb standing, sustained weight shifting, single-limb standing) and concluded that their findings supported the theory that standing posture is a dynamic event and that a small amount of postural sway is normal. Furthermore, they reported that the mean $\mathrm{COP}$ was $1.0 \pm 2.0 \mathrm{~cm}$ anterior to the tubercles of the naviculars and close to the midline of the base of support for all subjects, while the mean position of the COP fell slightly to the right of the midline for two thirds of the subjects and slightly to the left for one third of the subjects. The position did not relate to the side of the dominant limb. Distances between the mean positions during weight shifting in the four directions extended an average of $54 \%$ of the A $-\mathrm{P}$ dimensions and 59\% of the $\mathrm{M}-\mathrm{L}$ dimensions of the base of support. The youngest group, however, demonstrated the largest area of stability over which weight could be shifted and controlled. The oldest group demonstrated the smallest area. Finally, the authors reported very small mean values of postural sway for all subjects. Furthermore, tests performed by Arcan et al. (1) on normal subjects (free of known pathological conditions) indicated that subjects carried 45$65 \%$ of their body weight on their heels, $1-8 \%$ on their midfoot, and $30-45 \%$ on their forefoot. 
Riach and Hayes (49) answered some important questions about postural sway through the use of regression analyses, although they sampled only children (age 2-14). Most of their findings were consistent with previous research; however, addition of the variables sex, height, and weight to the multiple regression analysis failed to explain additional variance. Postural sway decreased linearly with age. Boys tended to become more stable at a faster rate than girls but started from an initially greater level of instability. Interestingly, the results revealed that eye closure had little effect on the children's postural stability. This obviously contradicts how research has described the Romberg test and supports the view that children use visual information to control balance differently than adults. The results suggest that vision is not as important a balance control strategy in children compared to adults until approximately age 7.

In general, the literature consistently reports that there is more $\mathrm{A}-\mathrm{P}$ sway than $\mathrm{M}-\mathrm{L}$ sway in normal subjects (12), that single-leg stances produce greater sway than double-leg stances (11, $12)$, and that eyes-closed trials produce greater sway than eyes-open trials $(11,12,51)$. Furthermore, older subjects demonstrate greater sway than younger subjects, with the exception of young children, who typically test poorly until adolescence $(11,12,36,51)$. Decreases in central nervous system control of postural muscle tone and balance result in decreased reflex patterns and increases in postural sway (25). It has also been proposed that the decreased steadiness in older subjects could be due to sensory inputs being processed less sensitively, and that in weight shifting trials, previous experience has influenced the central controlling mechanisms to avoid risks (36). Sugano and Takeya (51) suggested that increased body movement in older persons might be due to hypertension, arteriosclerosis, and muscle atrophy.

\section{Summary}

The clinical and research applications of balance assessment are endless. So much of what we do in rehabilitation is related to balance. Why shouldn't we begin to measure it? We are not suggesting that balance assessment should replace other important assessment techniques that clinicians use daily. It should, however, be considered as one ingredient in the decision-making process, especially in the cases of head injury and lower extremity musculoskeletal injury.

Balance is obviously influenced by a host of factors. Many of these factors are relevant to sport performance and should be considered in clinical decision making. Clinicians need to be aware of an athlete's history (symptoms and functional problems) as well as activity level and goals. It is important to account for extraneous variables before assessing a person's balance. The musculoskeletal system should always be evaluated first. Joint range of motion, muscle strength, endurance, and limitations due to pain can all lead to large compensatory hip and trunk motions to correct disequilibrium while standing $(27,56)$. Horak $(27)$ referred to this component as being biomechanical in nature. Another factor, according to Horak (27), is motor coordination, which involves an athlete's ability to make postural adjustments according to selective attention, reaction time, movement time, agility, and motivational arousal. Whitney (56) referred to the surrounding environment (i.e., terrain, participants, and spectators) as playing an important role in controlling balance. The final component, and possibly the most researched, is sensory organization. This involves one's ability to utilize somatosensory, vestibular, visual inputs in controlling balance. Research is becoming increasingly focused on identification of pathological conditions through balance assessment. Thus, such conditions obviously need to be considered before assessments are executed. 
Finally, there is definitely a need for more research focusing on functional validity of balance assessment in the sports medicine setting. While results of balance assessment have been correlated with functional capacity (i.e., falls, speed of gait, etc.) in the elderly, critics have questioned how these results correlate to the functional performance of trained athletes. Competition among manufacturers of the high-tech balance systems will hopefully reduce the cost of the units so they can find their way into more sports medicine settings for both clinical and research purposes.

\section{References}

1. Arcan, M., M. Brull, T. Najenson, and P. Solzi. FGP assessment of postural disorders during the process of rehabilitation. Scand. J. Rehabil. Med. 9:165-168, 1977.

2. Barret, D. Proprioception and function after anterior cruciate reconstruction. J. Bone Joint Surg. (Br.) 73:833-837, 1991.

3. Black, 0., C. Wall, H. Rockette, and R. Kitch. Normal subject postural sway during the Romberg test. Am. J. Otolaryngol. 3(5):309-318, 1982.

4. 4, Booher, J., and G. Thibodeau. Athletic Injury Assessment. St. Louis, MO: Times Mirror/Mosby College Publishing, 1995,

5. Cornwall, M., and P. Murrell. Postural sway following inversion sprain of the ankle, J. Am. Podiatr. Med. Assoc. 81:243-247, 1991.

6. Davies, G. The need for critical thinking in rehabilitation. J. Sport Rehabil. 4(1):122, 1995.

7. Diener, H., J. Dichgans, B. Guschlbauer, and H. San. The significance of proprioception on postural stabilization as assessed by ischemia. Brain Res. 296:103-109, 1984.

8. Dietz, V., G. Horstmarm, and W. Berger. Significance of proprioceptive mechanisms in the regulation of stance. Prog. Brain Res. 80:419-423, 1989.

9. Donahoe, B., D. Turner, and T. Worrell. The use of functional reach as a measurement of balance in healthy boys and girls ages 5-15. Phys. Ther. 73(6): S71, 1993.

10. Dornan, J., G. Fernie, and P. Holliday. Visual input: Its importance in the control of postural sway. Arch. Phys. Med, Rehabil. 59:586-591, 1978.

11. Ekdahl, C., G. Jarnlo, and S. Anderson. Standing balance in healthy subjects: Evaluation of a quantitative test battery on a force platform. Scand. Jr. Rehabil. Med, 21:187195, 1989.

12. Era, P., and E. Heilckinen. Postural sway during standing: Unexpected disturbance of balance in random samples of men of different ages. J. Gerontol. 40(3):287-295, 1985.

13. Faculjak, P., K. Firoozbakshsh, D. Wausher, and M. McGuire. Balance characteristics of normal and anterior cruciate ligament deficient knees. Phys. Ther, 73:S22, 1993.

14. Fisher, A., S. Wietlisbach, and J. Wilberger. Adult performance on three tests of equilibrium. Am. J. Occup. Ther, 42(1):30-35, 1988.

15. Flores, A. Objective measures of standing balance. Neurology Report-Am. Phys. Ther. Assoc. 16(1):17-21, 1992.

16. Freeman, M. Instability of the foot after injuries to the lateral ligament of the ankle. J. Bone Joint Surg. 47B:669-677, 1965.

17. Freeman, M., M. Dean, and I. Hanham, The etiology and prevention of functional instability of the foot. J. Bone Joint Surg. 47B:669-677, 1965. 
18. Gauffin, H., and H. Tropp. Altered movement and muscular-activation patterns during the one-legged jump in patients with old anterior cruciate ligament rupture. Am.. J. Sports Med. 20:182-192, 1992.

19. Goldie, P., T. Bach, and 0. Evans. Force platform measures for evaluating postural control: Reliability and validity. Arch. Phys. Med. Rehabil. 70:5 1 0-517, 1989.

20. Guskiewicz, K., and D. Perrin. Effect of Mild Head Injury on Postural Stability. 1995 (Abstract).

21. Guskiewicz, K., and Perrin. Effect of orthotics on postural sway following inversion ankle sprain. J. Orthop. Sport Phys. Ther. In review.

22. Guyton, A. Textbook of Medical Physiology (7th ed.). Philadelphia: Saunders, 1986.

23. Haaland, K., N. Temkin, G. Randahl, and S. Dikmen. Recovery of simple motor skills after head injury. J. Clin. Exp. Neuropsychol. 16(3):448-456, 1994.

24. Harrison, E., N. Duenkel, R. Dunlop, and G. Russell. Evaluation of single-leg standing following anterior cruciate ligament surgery and rehabilitation. Phys. Ther. 74(3):245252, 1994.

25. Hasselkus, B., and G. Shambes. Aging and postural sway in women. J. Gerontol. 30:661667, 1975.

26. Hellebrant, F., and G, Braun. The influence of sex and age on the postural sway of man. Am. J, Phys. Anthropol. 24(3):347-359, 1939.

27. Horak, F. Clinical measurement of postural control in adults. Phys. Ther. 67(12):1881$1885,1987$.

28. Horstmann, G., and V. Dietz. The contribution of vestibular input to the stabilization

29. of human posture: A new experimental approach. Neurosci. Lett. 95:179-184, 1988. 29. Ingersoll, C., and C. Armstrong. The effects of closed-head injury on postural sway. Med. Sci. Sports Exerc. 24(7):739-742, 1992.

30. Irrgang, J., S. Whitney, and E. Cox. Balance and proprioceptive training for rehabilitation of the lower extremity. J. Sport Rehabil. 3:68-83, 1994.

31. Jansen, E., R. Larsen, and B. Mogens. Quantitative Romberg's test: Measurement and computer calculations of postural stability. Acta Neural. Scand. 66:93-99, 1982.

32. Klonoff, P., L. Costa, and W. Snow. Predictors and indicators of quality of life in patients with closed-head injury. J. Clin. Exp. Neuropsychol. 8:469-485, 1986.

33. Lephart, S. Re-establishing proprioception, kinesthesia, joint position sense, and neuromuscular control in rehabilitation. In Rehabilitation Techniques in Sports (2nd ed.), W.E. Prentice (Ed.). St. Louis, MO: Times Mirror/Mosby College Publishing, 1993, pp. 118137.

34. Mauritz, K., J. Dichgans, and A. Hufschmidt. Quantitative analysis of stance in late cortical cerebellar atrophy of the anterior lobe and other forms of cerebellar ataxia, Brain 102:461-482, 1979.

35. Mizuta, H., M. Shiraishi, K. Kubota, K. Kai, and K. Takagi. A stabilometric technique for evaluation of functional instability in the anterior cruciate ligament deficient knee, Clin. .1. Sports Med. 2:235-239, 1992.

36. Murray, M., A. Seireg, and S. Sepic, Normal postural stability: Qualitative assessment. J. Bone Joint Surg. 57A(4):510-516, 1975.

37. Nashner, L. Adapting reflexes controlling the human posture. Exp. Brain Res. 26:5972, 1976. 
38. Nashner, L. Adaptation of human movement to altered environments. Trends Neurosci. 5:358-361, 1982.

39. Nashner, L. A functional approach to understanding spasticity. In Electromyography and Evoked Potentials, A. Struppler and A. Weindl (Eds.). Berlin: Springer-Verlag, 1985, pp. 22-29.

40. Nashner, L. Computerized dynamic posturography, In Handbook of Balance Function and Testing, G. Jacobson, C. Newman, and J. Kartush (Eds.). St. Louis, MO: Mosby Year Book, 1993, pp. 280-307.

41. Nashner, L. Practical biomechanics and physiology of balance. In Handbook of Balance Function and Testing, G. Jacobson, C. Newman, and J. Kartush (Eds.). St. Louis, MO: Mosby Year Book, 1993, pp. 261-279.

42. Nashner, L., and A. Berthoz. Visual contribution to rapid motor responses during postural control. Brain Res. 150:403-407, 1978.

43. Nashner, L., F. Black, and C. Wall, III. Adaptation to altered support and visual conditions during stance: Patients with vestibular deficits. J. Neurosci. 2(5):536-544, 1982.

44. Nashner, L., and G. McCollum. The organization of human postural movements: A formal basis and experimental synthesis. Behan. Brain Sci. 8:135-172, 1985.

45. Newton, R. Review of tests of standing balance abilities. Brain Injury 3:335-343, 1992.

46. Norre, M. Sensory interaction testing in platform posturography. J. Laryngol. Otol, 107:496-501, 1993.

47. Noyes, F., S. Barber, and R. Mangine. Abnormal lower limb symmetry determined by function hop test after anterior cruciate ligament rupture. Am. J. Sports Med. 19:516-518, 1991.

48. Orteza, L., W. Vogelbach, and C. Denegar. The effect of molded and unmolded orthotics on balance and pain while jogging following inversion ankle sprain. J. Athl, Train. 1:8084, 1992.

49. Riach, C., and K. Hayes. Maturation of postural sway in young children. Dev. Med. Clin. Neurol. 29:650-658, 1987.

50. Shumway-Cook, A., and F. Horak. Assessing the influence of sensory interaction on balance. Phys. Ther. 66(10):1548-1550, 1986.

51. Sugano, H., and T. Takeya. Measurement of body movement and its clinical applications. Jpn. J. Physiol. 20:296-308, 1970.

52. Taguchi, K. Relationship between the head's and the body's center of gravity during normal standing. Acta Otolaryngol. 90:100-105, 1980.

53. Tropp, H., J. Ekstrand, and J. Gillquist. Factors affecting stabilometry recordings of single limb stance. Am. J. Sports Med. 12:185-188, 1984.

54. Tropp, H., J. Ekstrand, and J. Gillquist. Stabilometry in functional instability of the ankle and its value in predicting injury. Med. Sci. Sports Exerc, 16:64-66, 1984.

55. Vander, A., J. Sherman, and D. Luciano. Human Physiology: The Mechanisms of Body Function (5th ed.). New York: McGraw-Hill, 1990.

56. Whitney, S. Clinical and High Tech Alternatives to Assessing Postural Sway in Athletes. Presented at the National Athletic Trainers' Association Annual Meeting, June 11, 1994, Dallas, TX. 